\title{
Multi-omics analysis of tumor mutational burden combined with prognostic assessment in epithelial ovarian cancer based on TCGA database
}

\author{
Jinhui Liu\#, Wei Xu\#, Siyue Li, Rui Sun, Wenjun Cheng ${ }^{\bowtie}$ \\ Department of Gynecology, the First Affiliated Hospital of Nanjing Medical University, Nanjing, Jiangsu Province, People's Republic of China. \\ \#These authors contributed equally to this article.
}

$\triangle$ Corresponding author: Wenjun Cheng, Department of Gynecology, the First Affiliated Hospital of Nanjing Medical University, Nanjing 210000, Jiangsu Province, People's Republic of China. Telephone: +86-25-86862863; E-mail: wenjunchengdoc@163.com.

(C) The author(s). This is an open access article distributed under the terms of the Creative Commons Attribution License (https://creativecommons.org/licenses/by/4.0/). See http://ivyspring.com/terms for full terms and conditions.

Received: 2020.07.09; Accepted: 2020.10.07; Published: 2020.10.23

\begin{abstract}
Background: Tumor mutation burden (TMB) is considered as a novel biomarker of response to immunotherapy and correlated with survival outcomes in various malignancies. Here, TMB-related genes (TRGs) expression signatures were constructed to investigate the association between TMB and prognosis in epithelial ovarian cancer (EOC), and the potential mechanism in immunoregulation was also explored.

Methods: Based on somatic mutation data of 436 EOC samples from The Cancer Genome Atlas database, we examined the relationship between TMB level and overall survival (OS), as well as disease-free survival (DFS). Next, the TRGs signatures were constructed and validated. Differential abundance of immune cell infiltration, expression levels of immunomodulators and functional enrichment in high- and low-risk groups were also analyzed.

Results: Higher TMB level revealed better OS and DFS, and correlated with earlier clinical stages in EOCs $(P=2.796 \mathrm{e}-04)$. The OS-related prognostic model constructed based on seven TRGs (B3GALTI, LIN7B, ANGPT2, D2HGDH, TAFI3, PFDN4 and DNAJC19) significantly stratified EOC patients into high- and low-risk groups $(P<0.001)$. The AUC values of the seven-gene prognostic signature at 1 year, 3 years, and 5 years were $0.703,0.758$ and 0.777 . While the DFS-related prognostic model was constructed based on the 4 TRGs (LPIN3, PXYLPI, IGSF23 and B3GALTI), with AUCs of $0.617,0.756$, and 0.731 , respectively. Functional analysis indicated that immune-related pathways were enriched in low-risk groups. When considering the infiltration patterns of immune cells, we found higher proportions of follicular helper $\mathrm{T}$ (Tfh) cell and MI macrophage, while lower infiltration of M0 macrophage in low-risk groups $(P<0.05)$. Accordingly, TMB levels of low-risk patients were significantly higher both in OS and DFS model $(P<$ $0.01)$.

Conclusions: Our TRGs-based models are reliable predictive tools for OS and DFS. High TMB may confer with an immunogenic microenvironment and predict favorable outcomes in EOCs.
\end{abstract}

Key words: epithelial ovarian cancer, tumor mutational burden, prognostic signature, immune infiltrates

\section{Introduction}

Epithelial ovarian cancer (EOC) is one of the leading causes of cancer death in women, with the 5 -year survival for all stages estimated at $45.6 \%$. This rate increases to more than $70 \%$ in the minority of patients who are diagnosed at an early stage, but declines to $35 \%$ in the vast majority of patients diagnosed at advanced stage [1]. Traditional surgery and adjuvant therapies have limited function to improve the prognosis of advanced EOC. Immunotherapy, especially treatment with immune checkpoint inhibitors has been a fast-moving field of clinical cancer research, and promising results of 
anti-PD-1-antibodies had been confirmed in recurrent EOC [2-4]. However, the reported overall response rate of anti-PD-1-antibodies was only 10.7\%-25\% [4-6]. Selecting patient groups that particularly benefit from immunotherapy is desiderated, but also clinically challenging as predictive biomarkers are lacking [7]. Further insights are desperately needed to predict the outcome of EOC to improve the survival rate.

Tumor mutational burden (TMB), the number of somatic mutations per DNA megabase $(\mathrm{Mb})$, has emerged as a novel biomarker of response to immunotherapy, with higher TMB inclining to harbor more neoantigens as targets for activated immune cells [8]. The positive relationship between TMB and response to CTLA-4 and PD-1 inhibition has been demonstrated in melanoma and non-small cell lung cancer $[9,10]$. There is growing evidence that ovarian cancers with a higher somatic mutation burden also respond better to cytotoxic chemotherapy [11]. Since the TMB scores observed in EOC are lower than that in other carcinomas, it remains unclear that whether patients with a relatively high TMB could benefit more from immunotherapy or have favorable survival outcomes.

In the present study (Fig S1), we investigated the relationship of TMB and prognosis of EOC using two TMB-related prognostic signatures, which were constructed with the somatic mutation and gene expression data from The Cancer Genome Atlas (TCGA). The independent prognostic power of that two models was confirmed by time-dependent receiver operating characteristic (ROC), Kaplan-Meier curve and multivariate Cox regression analysis. Furthermore, the differential abundance of immune cell infiltration, expression levels of immunomodulators and functional enrichment in high- and low-risk groups were also analyzed to understand the underlying mechanism in immunoregulation.

\section{Materials and methods}

\section{Data collection}

We downloaded the somatic mutation data from TCGA database via the GDC data portal (https:// portal.gdc.cancer.gov/), and selected the "Masked Somatic Mutation" data and processed it based on the VarScan software. We prepared the Mutation Annotation Format (MAF) of somatic variants and used "maftools" package in $\mathrm{R}$ software for the visualization and analysis of mutated spectral data. Besides, transcriptome profiles with HTSeq-FPKM format and clinical information including age, FIGO stage, tumor grade were all obtained from TCGA database (Table S1).

\section{Calculation of TMB and clinical data analysis}

To calculate TMB, the total number of mutations counted is divided by the size of coding region of the targeted territory. We divided the cases into high- and low-TMB group using the median as cut-off value. Then the TMB levels from TCGA cohort were merged with corresponding survival data of each sample via merge function in R. Kaplan-Meier analysis was conducted to compare the difference of OS and DFS between high- and low-TMB group. Correlations between TMB and clinical data were analyzed by Wilcoxon rank-sum test as well.

\section{Differentially expressed genes and pathway analysis}

The "limma" package was used to find the differentially expressed genes (DEGs) between highand low-TMB group. FDR $<0.05$ were taken as the cutoff criterion. Gene Ontology (GO) and Kyoto Encyclopedia of Genes and Genomes (KEGG) analysis of DEGs were implemented using "ggplot2" and "ClusterProfiler" packages.

\section{Construction and validation of TMB-related prognostic signatures}

OS and DFS were chosen as two important outcomes of EOC. Univariate Cox proportional hazards regression (CPHR) analysis was utilized to select the prognostic genes from TRGs with $P<0.05$. Candidate genes associated to a great extent with OS and DFS were assessed using Least Absolute Shrinkage and Selection Operator (LASSO) method, the coefficients and partial likelihood deviance were calculated with "glmnet" package in R. Multivariate CPHR analysis was further used to construct the prognostic signatures. Samples were classified into high- and low-risk group according to the median risk score, then, the survival difference was compared using the R package "survival". Time-dependent ROC curves were drawn to evaluate the predictive power of the prognostic signatures in both training and entire cohort by "timeROC" package. Moreover, a conjoint CPHR analysis was applied to define the independent prognostic variables of OS and DFS. Subgroup survival analyses stratified by clinicopathological factors were also conducted.

\section{Nomograms}

Prognostic nomograms that incorporated the TRGs signature and clinical risk factors were constructed by "rms" package to provide a quantitative method for individualized survival prediction. Prognostic accuracy of the nomograms at 1,3,5-years was compared and demonstrated by ROC and calibration curves. 


\section{Gene set enrichment analysis (GSEA)}

The collection of annotated gene sets of c2.cp.kegg.v6.0.symbols.gmt in Molecular Signatures Database (MSigDB, http://software.broadinstitute. org/gsea/msigdb/index.jsp) was chosen as the reference gene sets in GSEA software. $P<0.05$ were chosen as the cutoff criterion.

\section{Immune cell infiltration and immune checkpoints}

CIBERSORT is a newly developed algorithm for characterizing fractions of cell subsets using gene expression data obtained from bulk samples. We estimated the infiltration of 21 immune cell types (infiltration of naive CD4+ T cell was 0 in all samples) in low- and high-risk groups using CIBERSORT, with violin plots showing the distinct compositions. Then, we analyzed the relationship between 21 immune cell types and 17 crucial immune checkpoint modulators (including B7-H3, B7-H4, CD27, CD270, CD40, CD58, CD70, CD86, CTLA4, ICOS, IDO1, LAG3, PD-1, PD-L1, PD-L2, TIGIT, and TIM-3) in EOC sample [12-14]. The expression of immunomodulators in high- and low-risk group was also investigated.

\section{TMB profiles}

Wilcoxon rank-sum test was conducted to assess the TMB level of EOC samples in high- and low-risk groups. Mutation type, the top 10 mutated genes, associations across mutate genes in these groups were analyzed using "maftools" package.

\section{Statistical analysis}

Univariate, multivariate CPHR and LASSO analyses were executed to construct the TMB-related prognostic signatures, whose predictive capacities were evaluated using time-dependent ROC analysis. Conjoint CPHR analysis was applied to define the independent prognostic variables of OS and DFS, and the predictive accuracy of each variable was tested via time-dependent ROC analysis. Survival curves were estimated by Kaplan-Meier method and compared by the log-rank test. $P<0.05$ was determined statistically significant. All analyses were performed using R (version 3.3.1) and Bioconductor.

\section{Results}

\section{Genome-wide mutation profiling in EOC}

On the whole, various mutation categories were summarized in different groups, where missense mutation was the most common type. Singlenucleotide polymorphism occurred more frequently than deletion or insertion, and $\mathrm{C}>\mathrm{T}$ transition accounted for the largest part in the SNV classification. Horizontal histogram revealed the top
10 mutated genes in EOC, including TP53 (90\%), TTN (21\%), MUC16 (7\%), TOP2A (6\%), NF1 (6\%), CSMD3 (6\%), USH2A (6\%), RYR2 (5\%), HMCN1 (5\%) and FAT3 (5\%) (Fig. 1a). The coincident and exclusive associations across mutated genes were also investigated. For example, mutant MUC16 coexisted with mutant KMT2C, FLG, BRCA1, and APOB significantly $(P<0.05)$ (Fig. 1b). The waterfall plot showed details of mutation in 420 samples, where various colors with annotations at the bottom represented different mutation types (Fig. 1c).

\section{TMB correlated with survival outcomes and clinical stages}

After calculating the TMB value, we divided EOC patients into high- and low-TMB groups using the median TMB as cut-off value. Kaplan-Meier analysis with log-rank test indicated that patients in high-TMB group revealed better OS $(P=0.039)$ and DFS $(P=4.879 \mathrm{e}-04)$ than those in low-TMB group (Fig. $2 a, b)$. Higher TMB level also correlated with earlier clinical stages, while no significant difference was observed in associations with patient age or tumor grades (Fig. 2c-e).

\section{DEGs identification and functional analysis}

A total of 139 genes were identified as differentially expressed TRGs with FDR $<0.05$ (Fig S2a). Next, we conducted the GO enrichment analysis, which is composed of three parts: biological process (BP), cellular component (CC), and molecular function (MF). In BP group, regulation of chromosome segregation, ribosomal subunit export from nucleus and maintenance of cell polarity were enriched. In CC group, these DEGs were mainly involved in mitochondrial inner membrane, chromosome region. In MF group, the significantly enriched terms were cytoskeletal adaptor activity, DNA replication origin binding and exo-alphasialidase activity (Fig. S2b-d). Besides, KEGG signaling pathway analysis suggested that cell cycle was the most significant pathway (Fig. S2e).

\section{Development and assessment of the TMB- related prognostic signatures}

We integrated the transcriptome and clinical data so as to screen out 351 OS-related and 319 DFS-related prognostic EOC samples. For OS, training cohort composed of 176 randomly-selected samples were used to construct the prognostic signature, while the entire cohort was used to validate the predictive power. Similarly, the DFS signature was constructed based on 160 samples. After step-by-step gene selection through univariate CPHR, LASSO regression and multivariate $\mathrm{CPHR}$ analysis, finally, 
seven genes (B3GALT1, LIN7B, ANGPT2, D2HGDH, TAF13, PFDN4 and DNAJC19) were confirmed as the most OS-related genes, and the four genes (LPIN3, PXYLP1, IGSF23 and B3GALT1) were identified as the most DFS-related genes for EOC patients. Risk score for OS $=0.17849 *$ B3GALT1 $+(-0.15850) *$ LIN7B + $(-0.20095) * A N G P T 2+0.06441$ * D2HGDH $+(-0.03555)$ * TAF13 + $0.03600 *$ PFDN4 $+(-0.05400) *$ DNAJC19. Risk score for DFS $=0.06416$ * LPIN3 + $(-0.20524)$ * PXYLP1 + (-0.09458) * IGSF23 + 0.18717 * B3GALT1 (Table 1,2,3,4).

Table 1. Univariate cox analysis of TMB-related signature with OS

\begin{tabular}{llll}
\hline Gene & HR & $95 \% \mathrm{CI}$ & $p$ value \\
\hline ACSS3 & 1.422 & $1.173-1.723$ & 0.000 \\
ANGPT2 & 0.782 & $0.654-0.935$ & 0.007 \\
HAPLN1 & 0.622 & $0.427-0.904$ & 0.013 \\
D2HGDH & 1.065 & $1.012-1.120$ & 0.016 \\
TAF13 & 0.962 & $0.931-0.993$ & 0.018 \\
RARG & 1.024 & $1.003-1.045$ & 0.024 \\
LPCAT4 & 1.046 & $1.006-1.089$ & 0.025 \\
DNAJC19 & 0.964 & $0.932-0.996$ & 0.029 \\
PFDN4 & 1.018 & $1.002-1.034$ & 0.030 \\
B3GALT1 & 1.160 & $1.009-1.333$ & 0.037 \\
PGM3 & 0.907 & $0.825-0.997$ & 0.043 \\
SELENOT & 0.986 & $0.973-1.000$ & 0.044 \\
GCH1 & 0.886 & $0.786-0.998$ & 0.046 \\
LIN7B & 0.817 & $0.669-0.997$ & 0.047 \\
LTA4H & 1.029 & $1.000-1.059$ & 0.050 \\
\hline
\end{tabular}

Table 2. Multivariate Cox analysis of TMB-related signature with OS

\begin{tabular}{|c|c|c|c|c|}
\hline Gene & description & coef & HR & $p$ value \\
\hline B3GALT1 & $\begin{array}{l}\text { Beta-1,3-galactosyltransferase } \\
1\end{array}$ & 0.17849 & $1.20(1.04-1.37)$ & 0.01026 \\
\hline LIN7B & Lin-7 homolog B & -0.15850 & $0.85(0.69-1.05)$ & 0.13512 \\
\hline ANGPT2 & Angiopoietin 2 & -0.20095 & $0.82(0.69-0.98)$ & 0.02504 \\
\hline D2HGDH & $\begin{array}{l}\text { D-2-hydroxyglutarate } \\
\text { dehydrogenase }\end{array}$ & 0.06441 & $1.07(1.01-1.13)$ & 0.02468 \\
\hline TAF13 & $\begin{array}{l}\text { TATA-box binding protein } \\
\text { associated factor } 13\end{array}$ & -0.03555 & 0.97(0.93-1.00) & 0.05265 \\
\hline PFDN4 & Prefoldin subunit 4 & 0.03600 & $1.04(1.02-1.05)$ & 0.00001 \\
\hline DNAJC19 & $\begin{array}{l}\text { DnaJ heat shock protein } \\
\text { family (Hsp40) member C19 }\end{array}$ & -0.05400 & $0.95(0.91-0.98)$ & 0.00482 \\
\hline
\end{tabular}

In training cohort, we calculated the seven-gene based risk score for each patient, and then separated the samples into high- $(\mathrm{n}=88)$ and low-risk group $(n=88)$ using the median value as cutoff point. Patients in entire cohort were also separated into high- $(\mathrm{n}=174)$ and low-risk group $(\mathrm{n}=177)$ according to the same cutoff value. The distribution of risk scores, OS, OS status, and expression of seven genes in the cohorts were showed in Fig. 3a-c. Kapan-Meier survival curves of the training and entire cohort suggested the same result: EOC patients in the low-risk group had much better OS than those in the high-risk group $(P<0.001)$. To assess the predictive performance of the seven-gene based signature, we conducted a time-dependent ROC curve analysis by comparing the respective AUC value. The AUC values of the seven-gene prognostic signature at 1 year, 3 years, and 5 years were $0.703,0.758$ and 0.777 in the training cohort, while $0.666,0.666,0.645$ in the entire cohort, accordingly (Fig. 3d).

Table 3. Univariate cox analysis of TMB-related signature with DFS

\begin{tabular}{llll}
\hline Gene & HR & $95 \%$ CI & $p$ value \\
\hline PXYLP1 & 0.832 & $0.724-0.956$ & 0.009 \\
LPIN3 & 1.065 & $1.013-1.120$ & 0.014 \\
GCH1 & 0.862 & $0.763-0.973$ & 0.016 \\
B3GALT1 & 1.182 & $1.029-1.356$ & 0.018 \\
CDC6 & 0.884 & $0.796-0.982$ & 0.021 \\
C3orf38 & 0.921 & $0.855-0.993$ & 0.032 \\
NOCT & 0.846 & $0.724-0.988$ & 0.035 \\
RASSF7 & 1.008 & $1.001-1.015$ & 0.036 \\
IGSF23 & 0.913 & $0.837-0.996$ & 0.041 \\
TRIP6 & 1.010 & $1.000-1.020$ & 0.045 \\
\hline
\end{tabular}

Table 4. Multivariate Cox analysis of TMB-related signature with DFS

\begin{tabular}{|c|c|c|c|c|}
\hline Gene & description & coef & HR & $P$ value \\
\hline IGSF23 & $\begin{array}{l}\text { Immunoglobulin } \\
\text { superfamily member } 23\end{array}$ & -0.09458 & $0.91(0.83-0.99)$ & 0.03392 \\
\hline LPIN3 & Lipin 3 & 0.06416 & $1.07(1.01-1.12)$ & 0.01644 \\
\hline PXYLP1 & $\begin{array}{l}\text { 2-phosphoxylose } \\
\text { phosphatase } 1\end{array}$ & -0.20524 & $0.81(0.70-0.94)$ & 0.00612 \\
\hline B3GALT1 & $\begin{array}{l}\text { Beta-1,3-galactosyltransferase } \\
1\end{array}$ & 0.18717 & $1.21(1.04-1.39)$ & 0.01107 \\
\hline
\end{tabular}

As for DFS model, patients in training cohort were allocated into high- $(\mathrm{n}=80)$ and low-risk group $(n=80)$ according to the median value calculated based on the four-gene prognostic signature, with the entire cohort grouping into high- $(n=144)$ and low-risk $(n=175)$. Distribution of risk score, DFS, DFS status, and prognostic-gene expression were showed in Fig S3a-c. Kapan-Meier survival curves showed patients with low risk scores had longer DFS in both cohorts $(P$ $<0.01$ ). AUC values of the four-gene prognostic signature at 1 year, 3 years, and 5 years were 0.617 , 0.756 , and 0.731 in the training cohort, while 0.608 , $0.670,0.778$ in the entire cohort, respectively (Fig. S3d).

Furthermore, we performed subgroup analysis of the two signatures in age $(\leq 60,>60)$, tumor grade (G1 \& G2, G3 \& G4) and clinical stage (stage I \& II, stage III \& IV), which suggested that patients with high-risk scores had shorter OS and DFS in subgroups of age $\leq 60$, age $>60$, G3 \& G4 and stage III \& IV ( $P$ $<0.05$ ) (Fig S4). Heatmaps showed expression profiles of the prognosis-related genes in high- and low-risk groups. In OS model, the differential expression was significantly associated with age, while no significant 
association was found in DFS model (Fig S5).

To assess whether the signature was an independent predictor of EOC, we analyzed the relationships between $O S$ and clinical factors by CPHR model. Multivariate analysis showed that only the signature-based risk score was significantly associated with OS $(P<0.05)$ (Table S2). Risk score was also proved to be an independent prognostic predictor of DFS (Table S3).

In order to establish a clinically applicable method for predicting the survival probability of EOC patients, nomograms including the signature-based

a
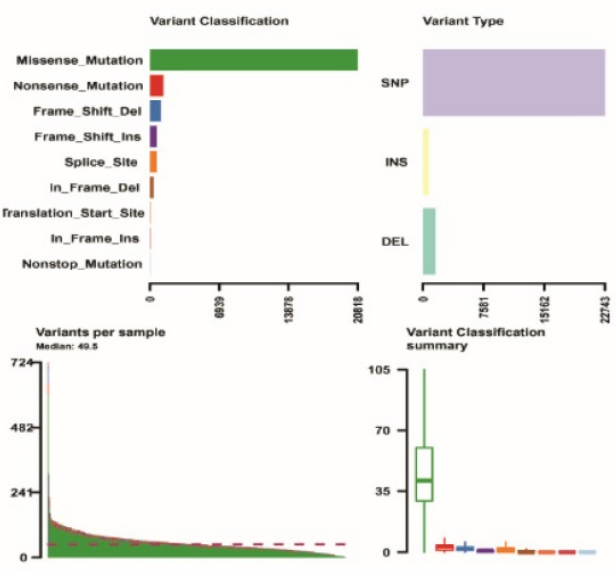
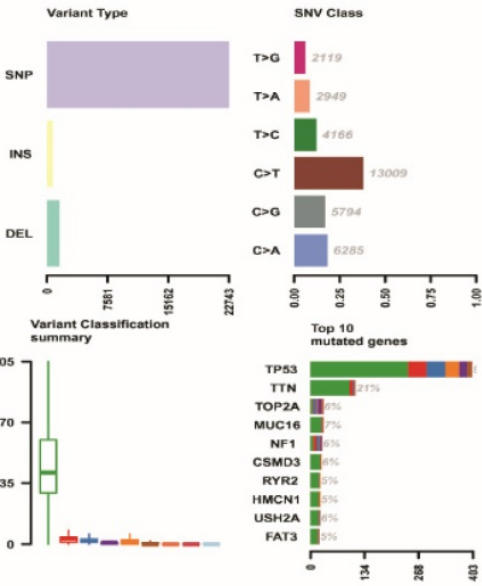

risk score and clinical factors were developed (Fig. 4a, Fig S6a). By drawing time-ROC curves, we found that the gene-based signatures had better predictive ability for 1 year-, 3 years-, 5 years-OS and DFS as compared with other clinical factors. Intriguingly, when combining risk score with clinical factors for analysis, the AUC values of 1 year-, 3 years-OS and DFS increased further (Fig. 4b-d, Fig S6b-d), which suggested the nomograms had superior predictive capacity for short-term prognosis. Calibration plots also verified good calibration ability of the signature-based nomograms (Fig. 4e-g, Fig S6e-g). b

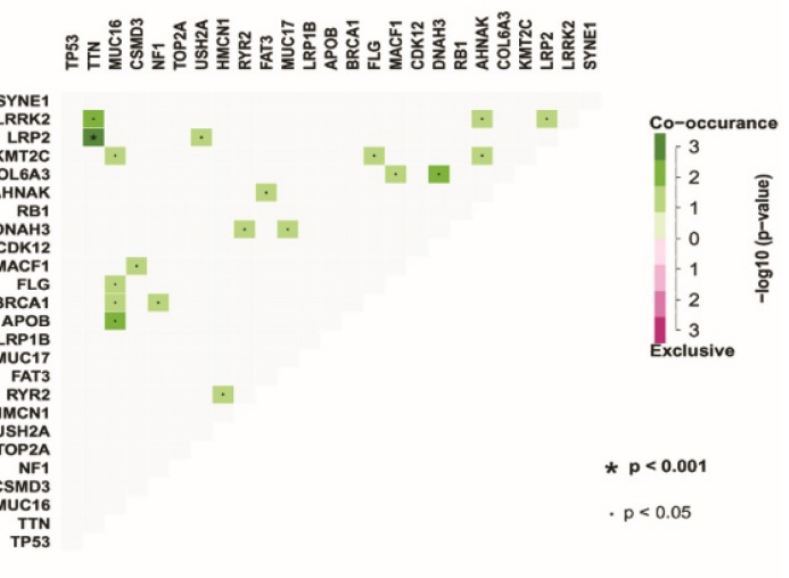

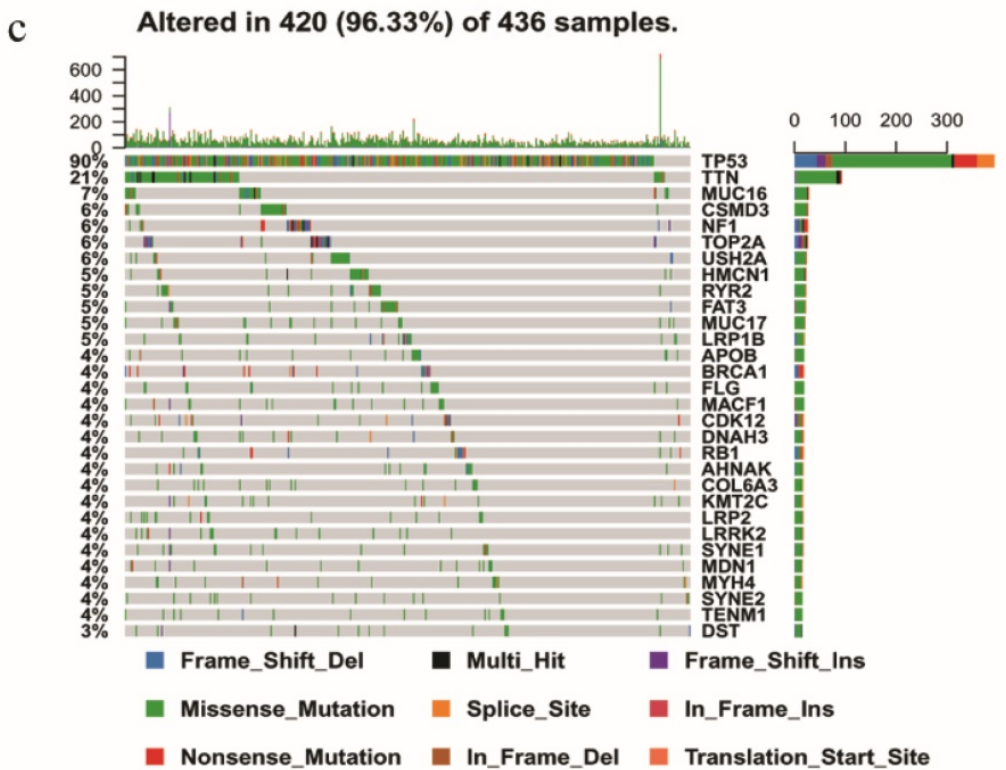

Figure 1. Summary of mutation profiling in EOC samples. a Distribution of variants based on variant classification, type, and SNV class. Bottom part (from left to right) indicates mutation load for each sample, and the top 10 mutated genes in EOC. $\mathbf{b}$ The coincident and exclusive associations across mutated genes displayed as a triangular matrix. c Landscape of mutation profiles was shown in the waterfall plot, in which the type of mutation is shown in the comment bar (bottom) and genes are ordered by their mutation frequency. 
a

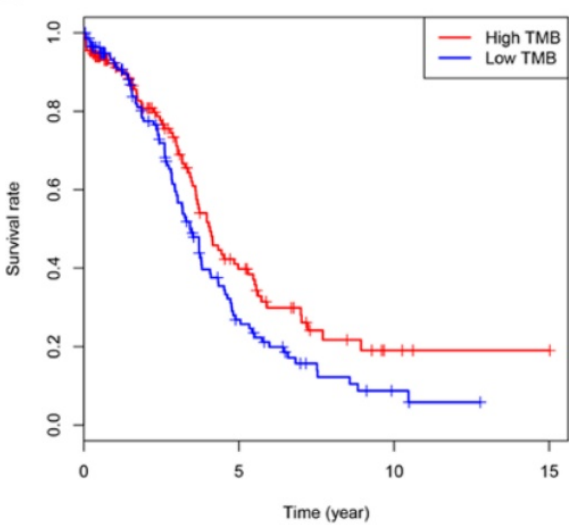

b

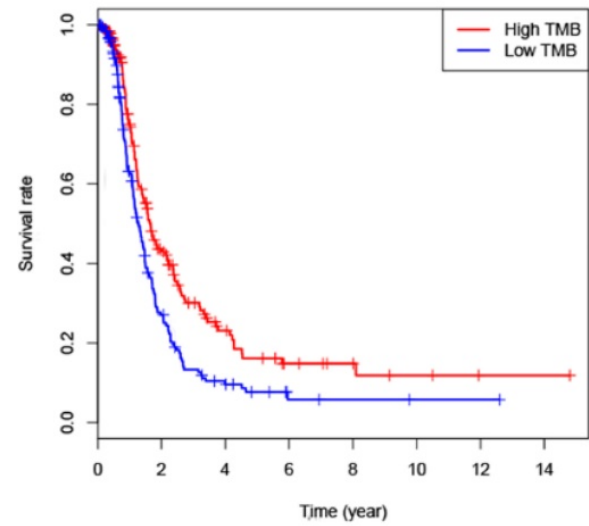

C

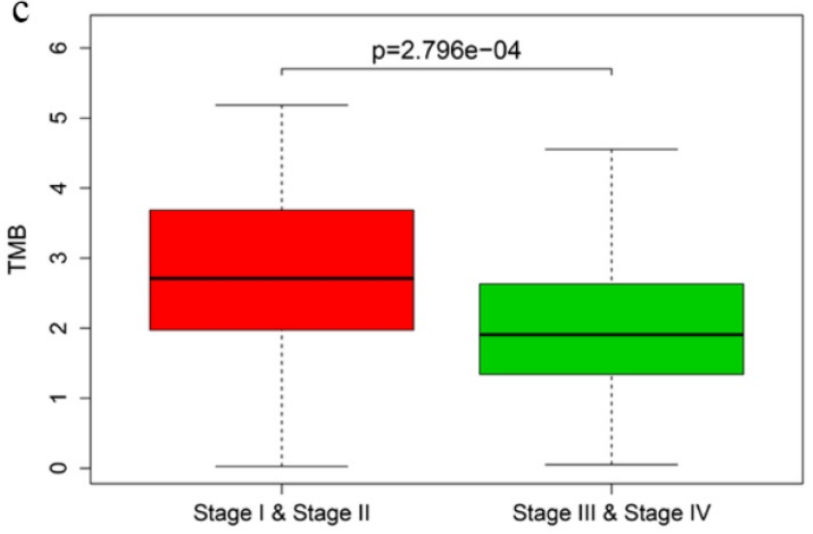

d

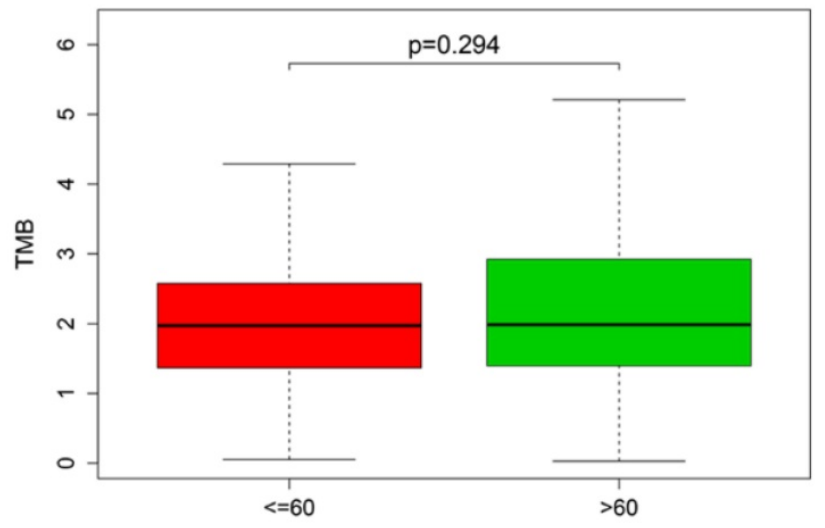

e

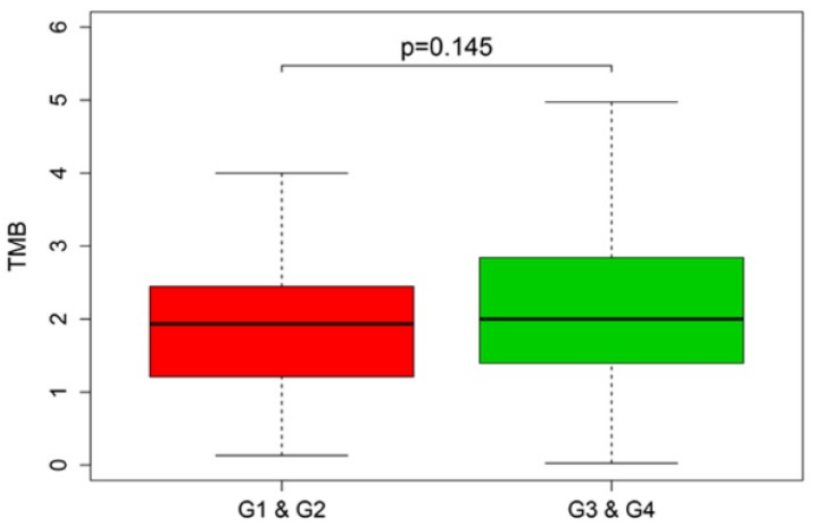

Figure 2. Prognosis of TMB and associations with clinical characteristics. a, b Higher TMB level revealed better OS $(P=0.039)$ and DFS $(P<0.001)$. c-e Higher TMB level correlated with earlier clinical stages with $\mathrm{p}=2.796 \mathrm{e}-04$, while no significant difference was observed in patient age or tumor grades.

\section{Identification of the prognostic signatures related biological pathways}

By comparing the biological processes enriched in high- and low-risk groups, we defined the underlying biological function of the prognostic genes. In OS model, gene sets for "estrogen response", "hedgehog signaling", "mitotic spindle", "myogenesis" and "Wnt/ $\beta$-catenin signaling" were enriched in high-risk group (Fig. 5a), while "allograft rejection", "IL-6-JAK-STAT3 signaling”, "interferon response" and "oxidative phosphorylation" pathways were enriched in low-risk group (Fig. 5b). As for DFS model, gene sets for "coagulation", "epithelial mesenchymal translation", "myogenesis" and "TNFA signaling via NF-kB" were enriched in high-risk group (Fig. 5c), while "interferon response", "oxidative phosphorylation" and "reactive oxygen species pathway" were enriched in low-risk group (Fig. 5d). The GSEA results demonstrated that immune-related pathways were enriched in low-risk groups. Therefore, we suggested that the TMB-related 
risk signatures may demonstrate an intensive immune phenotype.

\section{Differential immune cells infiltration in high- and low-risk groups}

Most solid tumors are infiltrated by myeloidand lymphoid lineage-derived immune cells that are differentially distributed within the tumor microenvironment (TME) with a crucial role in the establishment of antitumoral responses or tumor progression [15]. Thus, composition profiles of immune cells in high- and low-risk samples were identified and shown by the violin plots. Compared with the high-risk group (divided by OS-signature), samples in low-risk group generally contained higher proportions of follicular helper $\mathrm{T}(\mathrm{Tfh})$ cell, $\gamma \delta \mathrm{T}$ cell and M1 macrophage, but lower proportions of regulatory $\mathrm{T}$ cell $(\mathrm{T}$-reg) and resting mast cell $(P<$ 0.05 ) (Fig. 6a). As to DFS-signature, infiltrating levels of plasma cell, Tfh cell, $\gamma \delta$ T cell and M1 macrophage were higher in low-risk group, while that of M0 macrophage was lower $(P<0.05)$ (Fig. 6b). Furthermore, Tfh cell and M1 macrophage correlated with the survival rate positively, while M0 macrophage did negatively (Fig. 7a-c). train set
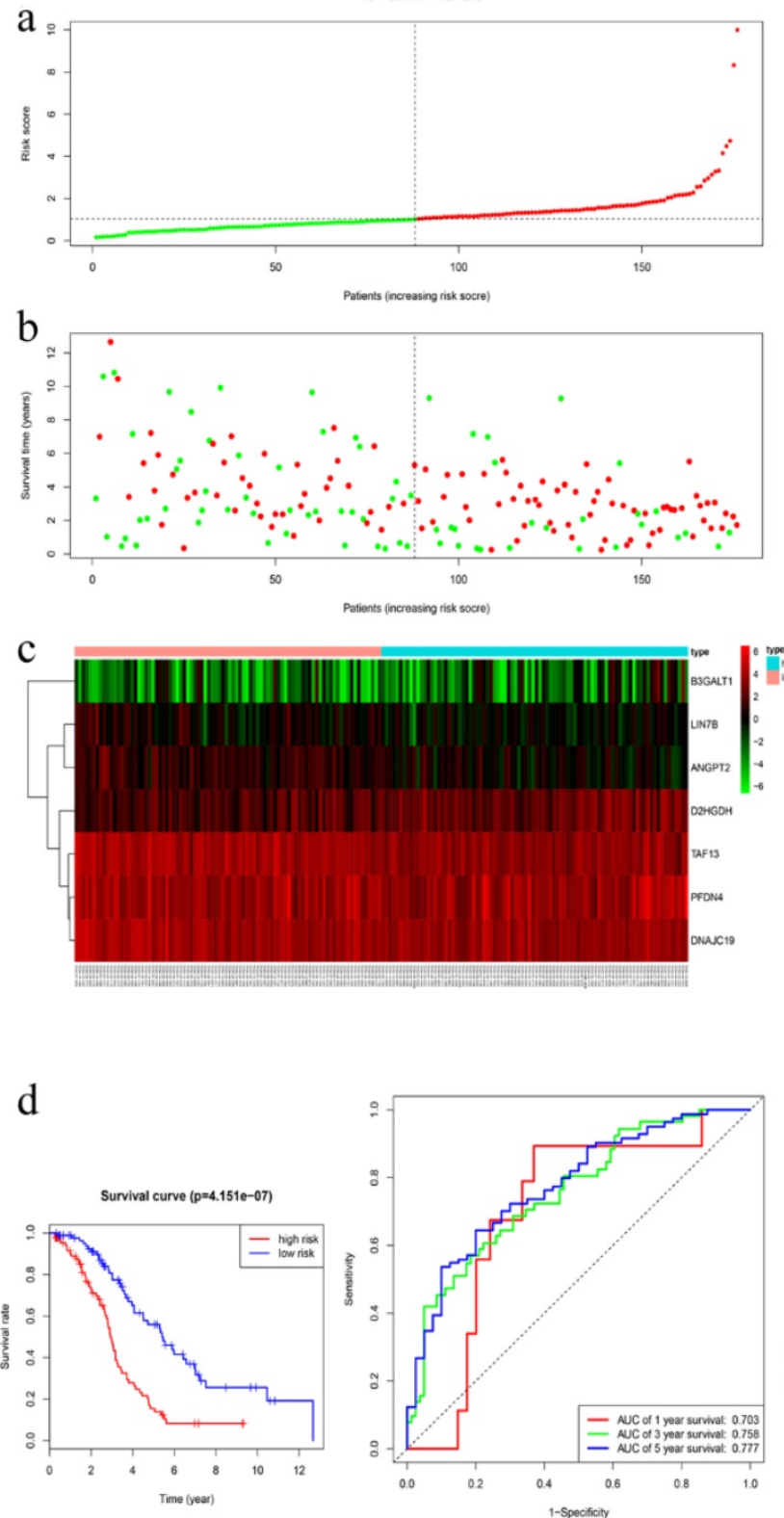

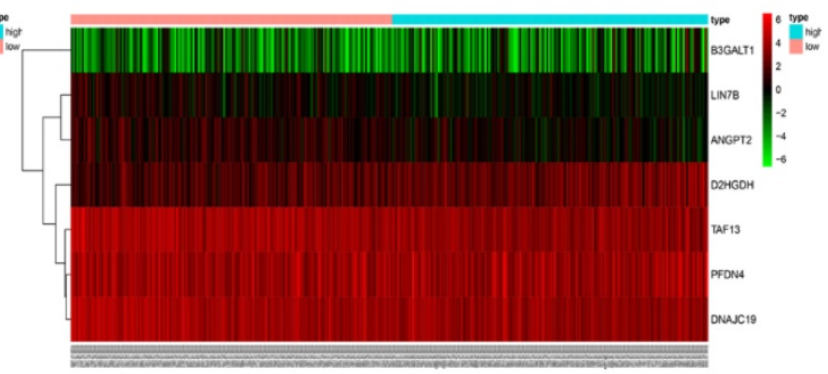

entire set
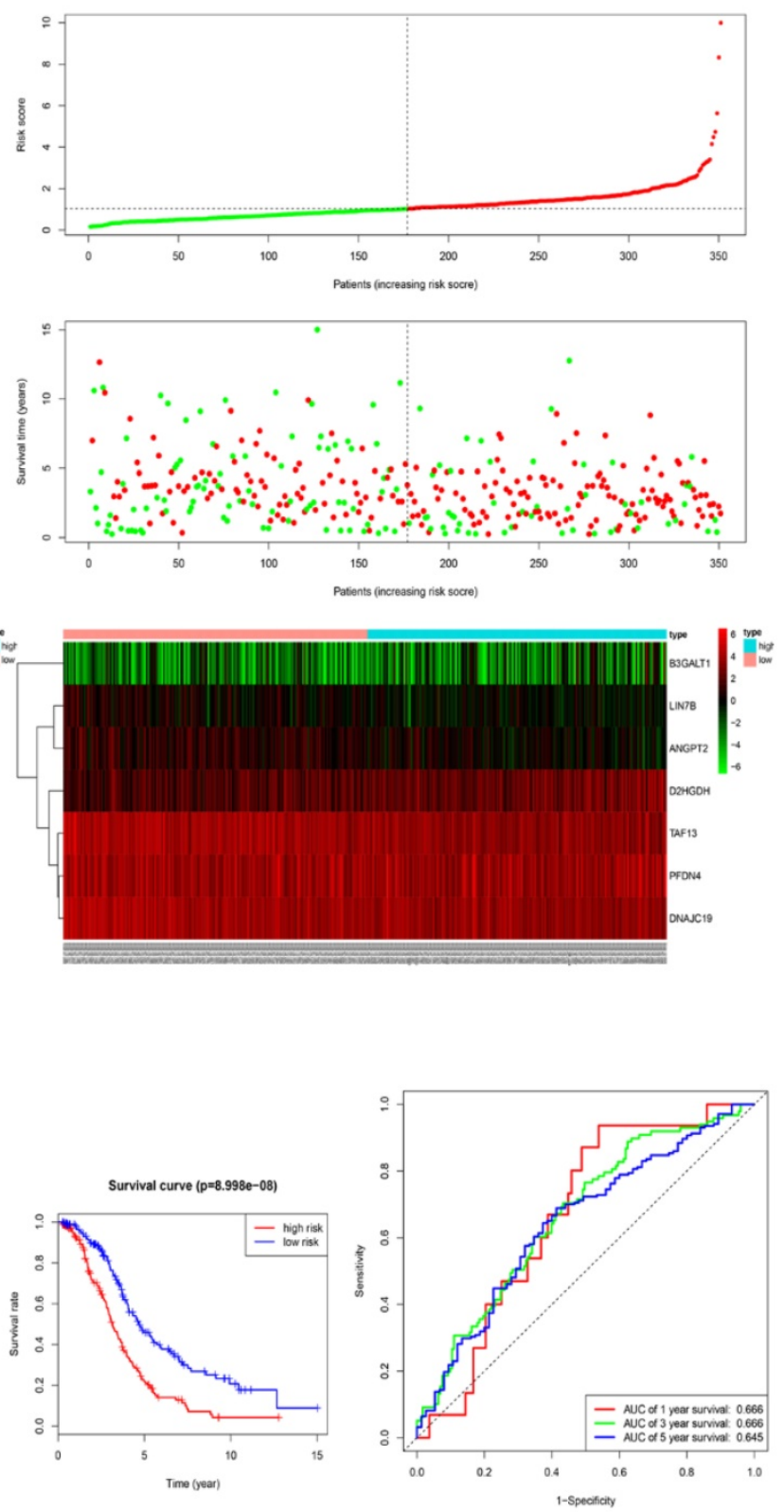

Figure 3. Characteristics of the 7-gene prognostic signature in the training and entire cohort. a The risk score of each EOC patient; $\mathbf{b}$ OS and survival status of the patients; $\mathbf{c}$ Heat maps of gene expression profiles; $\mathbf{d}$ Left panel: Kapan-Meier curves suggested thar EOC patients in low-risk group had much better OS than those in the high-risk group ( $P$ $<0.001)$. d Right panel: Time-dependent ROC curves at 1 year, 3 years and 5 years based on the7-gene signature. 
a

Poims

$\infty \quad+\infty$

cost

$-$

$-$

$=$

b

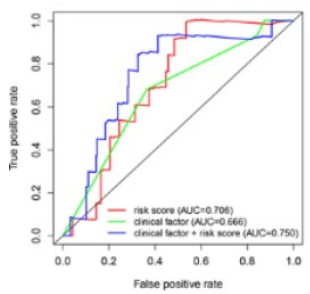

\section{C}

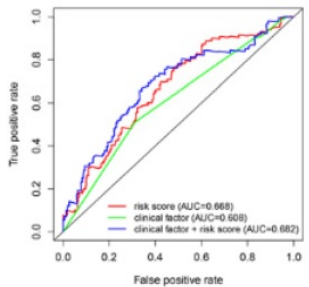

d

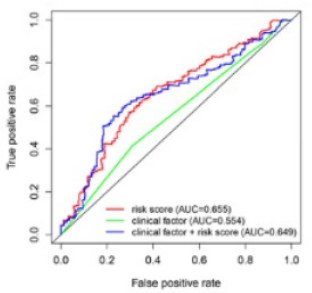

e

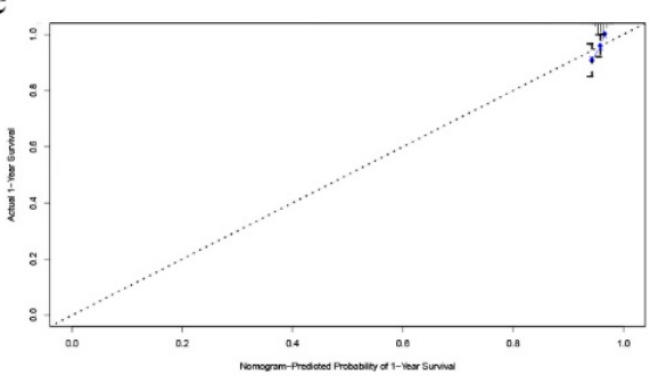

$\mathrm{f}$
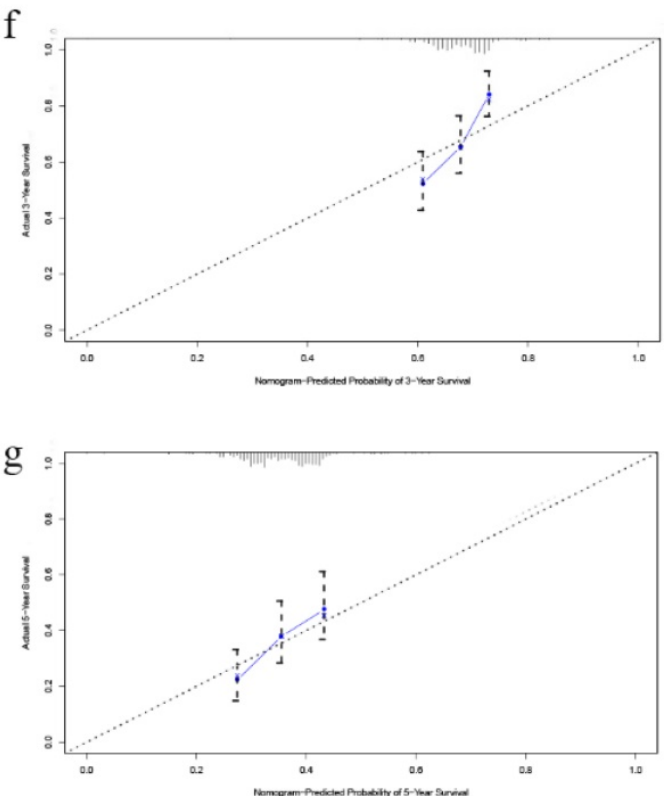

Figure 4. Nomogram to predict OS in EOC patients. a Nomogram based on the 7-gene signature and clinical factors for 1-, 3-and 5-year OS prediction. b Time-dependent ROCs for the nomogram, clinic factors including age, tumor grade and clinical stage. c Calibration plots of the gene-based prognostic model.

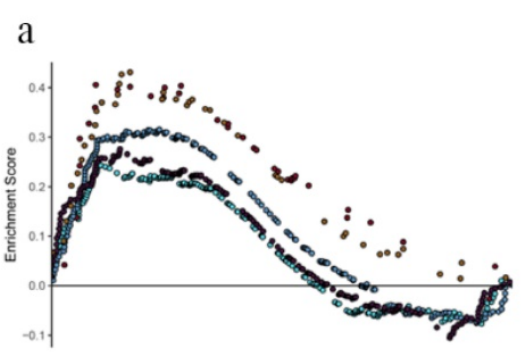

- HALMMRR_ESTROGEN_RESPONSE_EARUY

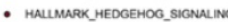

- HALUMARK_Mitotic_SPRINOLE

MR. MMOGENESS

- maumark_mat aeta_catenn_signaung

\section{b}
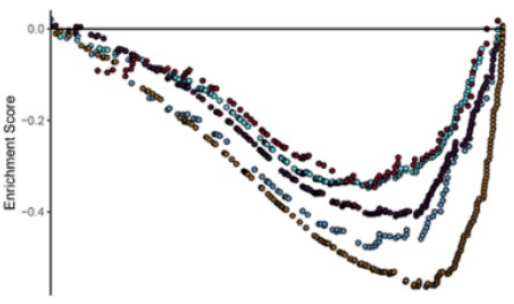

- hollmark_allograft_REJection

- haumarónterseron

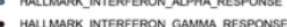

- hulumark_oxidative Phosphoprition.

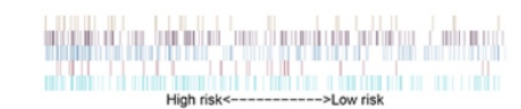

C
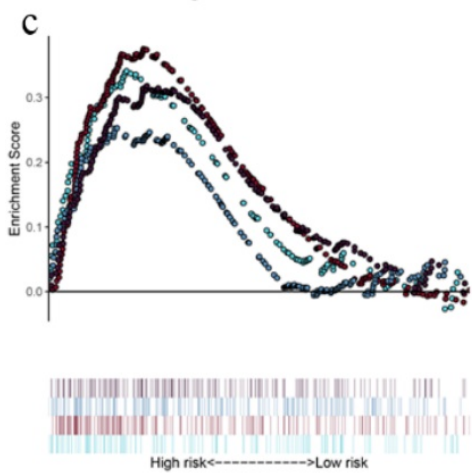

- haumaracongunator

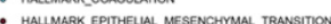
- raumer armectacs

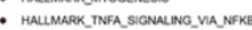

Figure 5. Functional enrichment of the prognostic genes of OS and DFS with GSEA. Pathway enriched in high-risk group of OS (a), low-risk group of OS (b), high-risk group of DFS (c), and low-risk group of DFS (d). $P<0.05$. 

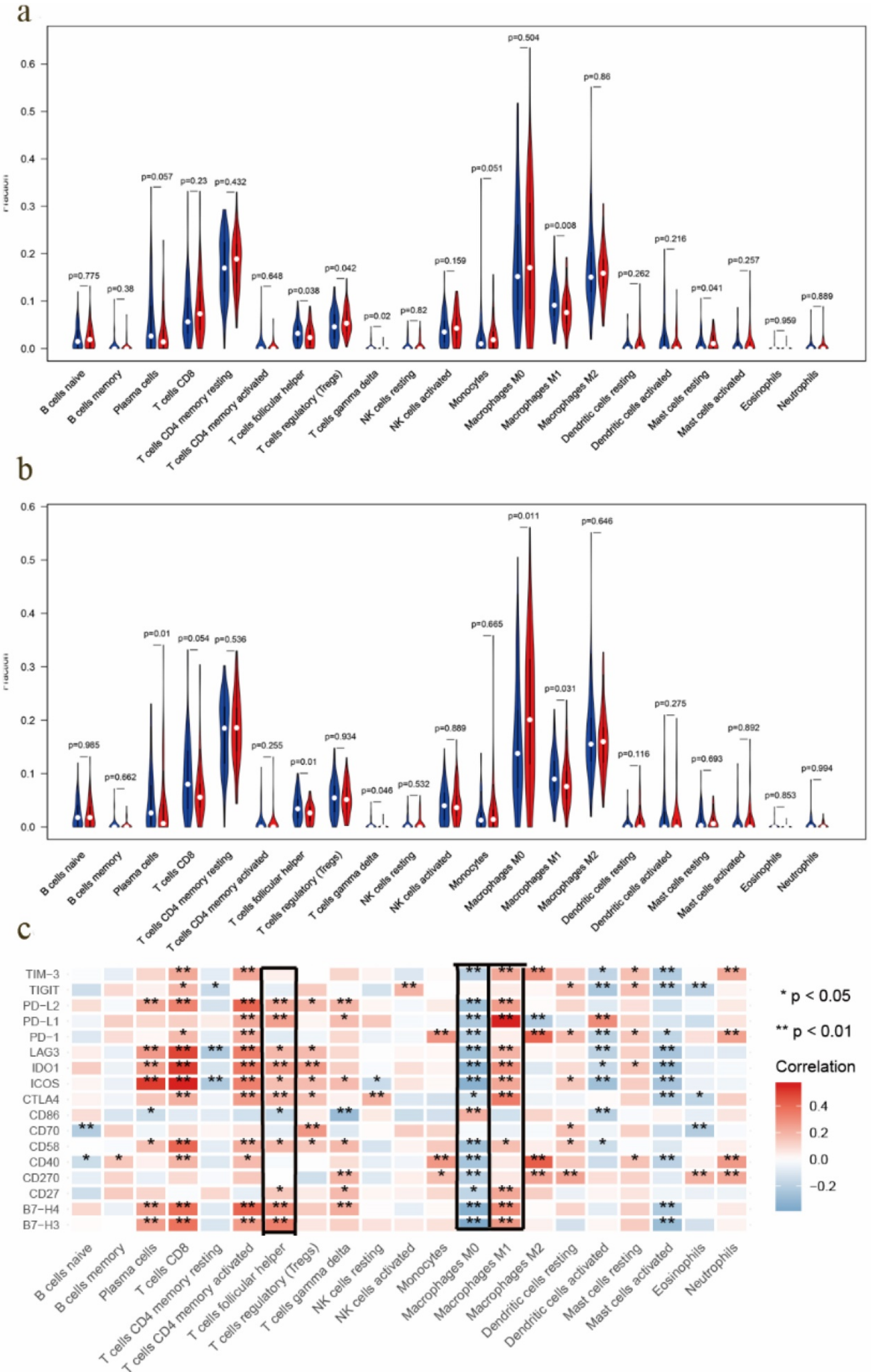

Figure 6. TIC distribution and the correlation between immunomodulators. a EOC samples in low-risk group (based on the OS-signature) had higher proportions of follicular helper T (Tfh) cell, $\gamma \delta \mathrm{T}$ cell and Ml macrophage, but lower proportions of T-reg and resting mast cell $(P<0.05)$. b Samples in low-risk group (based on the DFS-signature) had higher proportions of plasma cell, Tfh cell, $\gamma \delta$ T cell and Ml macrophage, but lower proportion of M0 macrophage $(P<0.05)$. c Correlations between immunomodulators and TICs, which showed Tfh cell and MI macrophage correlated positively with most modulators, while M0 macrophage did negatively. 

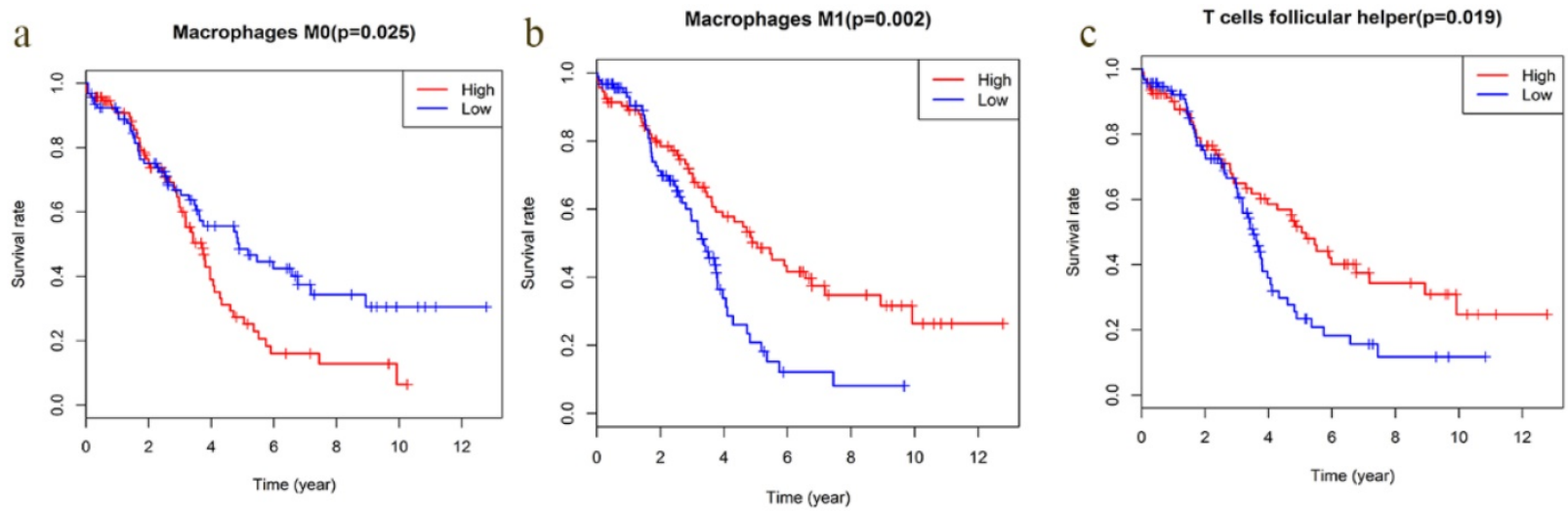

d ${ }_{87 . H 4}->11.09(245)-<11.09(106)$
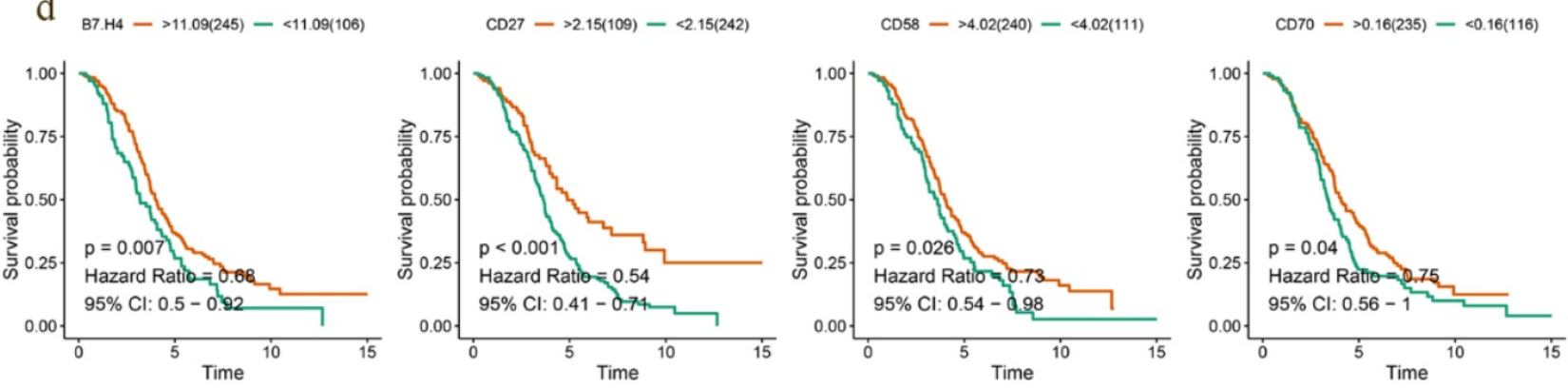

$1001->8.77(106)-<8.77(245)$

$\operatorname{lcos}->0.18(195)-<0.18(156)$

LAG3 $->2.39(139)-<2.39(212)$
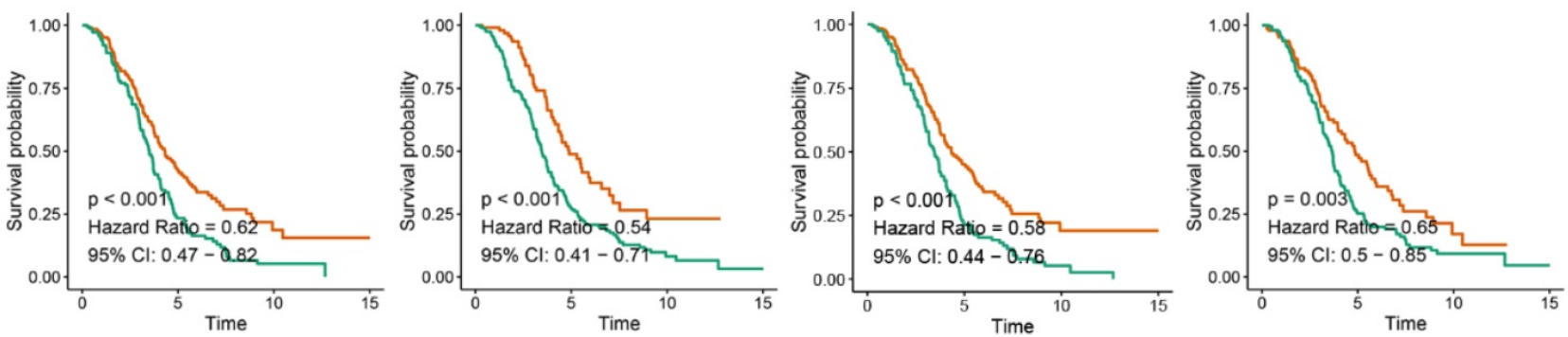

PD. 1 - >0.39(215) $-<0.39(136)$

PD.L1 $->0.43(236)-<0.43(115)$

PD.L2 $->0.7(201)-<0.7(150)$

TIGIT $->0.21(173)-<0.21(178)$
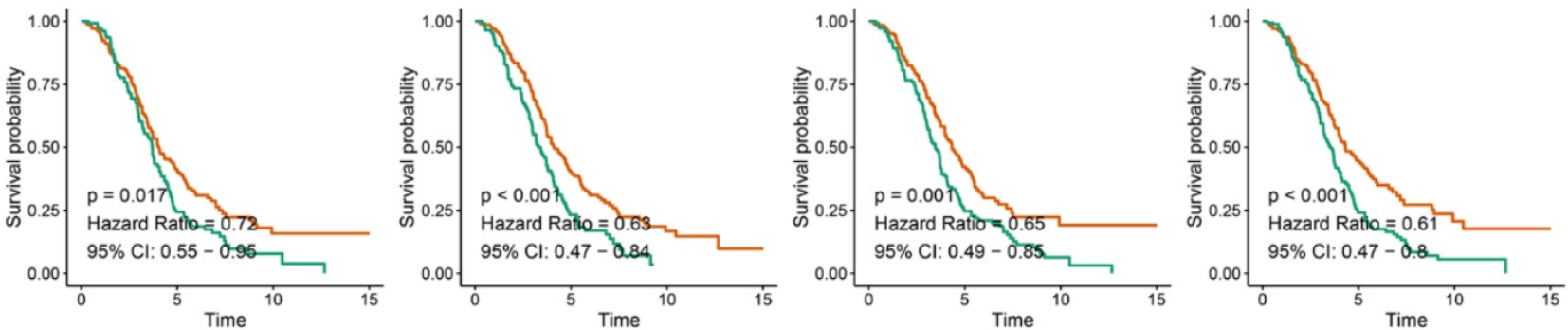

Figure 7. Survival analysis of TICs and immunomodulators. a-c Kaplan-Meier curves showed that Tfh cell and MI macrophage correlated with favorable survival outcome, but M0 macrophage correlated with poor survival in EOC. $\mathbf{d}$ High expression of B7-H4, CD27, CD58, CD70, CTLA4, ICOS, IDO1, LAG3, PDI, PD-LI, PD-L2 and TIGIT was shown to be associated with favorable survival outcome $(P<0.05)$.

\section{Prognostic value of immune checkpoint modulators and the correlation with immune cells}

Previous studies have discovered that immune checkpoint modulators mediate the function of tumor infiltrating cells [16]. Therefore, we evaluated the correlation of immunomodulators and immune cells in EOC, which suggested that Tfh cell and M1 macrophage correlated positively with most modulators, and M0 macrophage did negatively (Fig. $6 \mathrm{c}$ ). We also assessed the predictive performance of immunomodulators in EOC. Kaplan Meier curves demonstrated that high expression of B7-H4, CD27, CD58, CD70, CTLA4, ICOS, IDO1, LAG3, PD1, PD-L1, PD-L2 and TIGIT was associated with favorable survival significantly (Fig. 7d). Meanwhile, the differential expression profiles of immunomodulators indicated an immunogenic TME in low-risk EOC samples (Fig S7). 

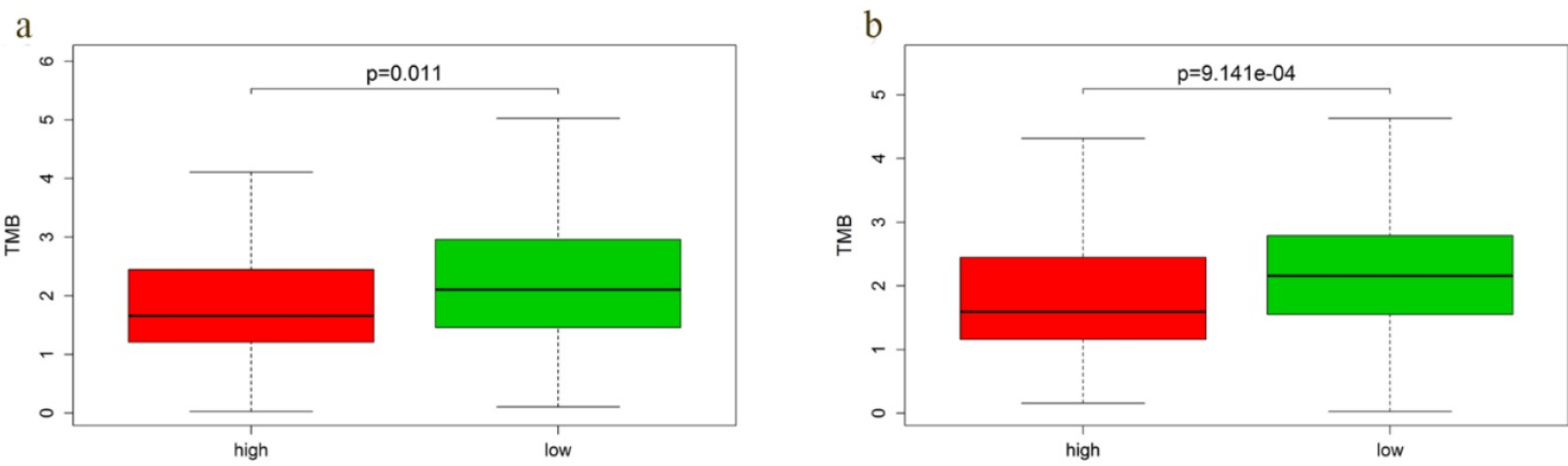

C

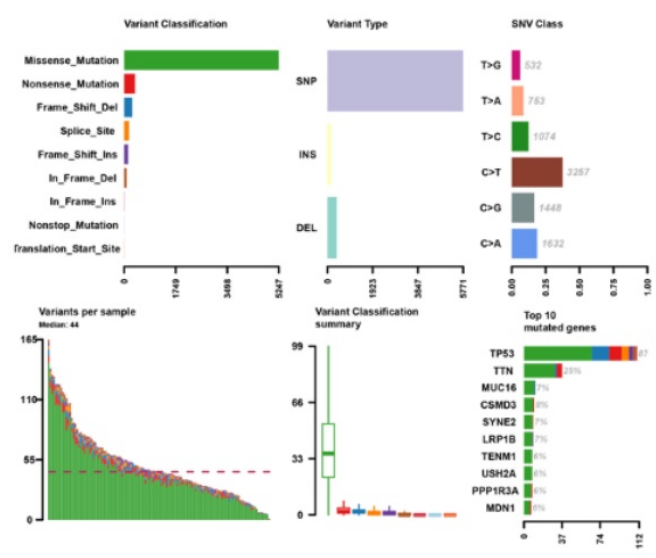

e
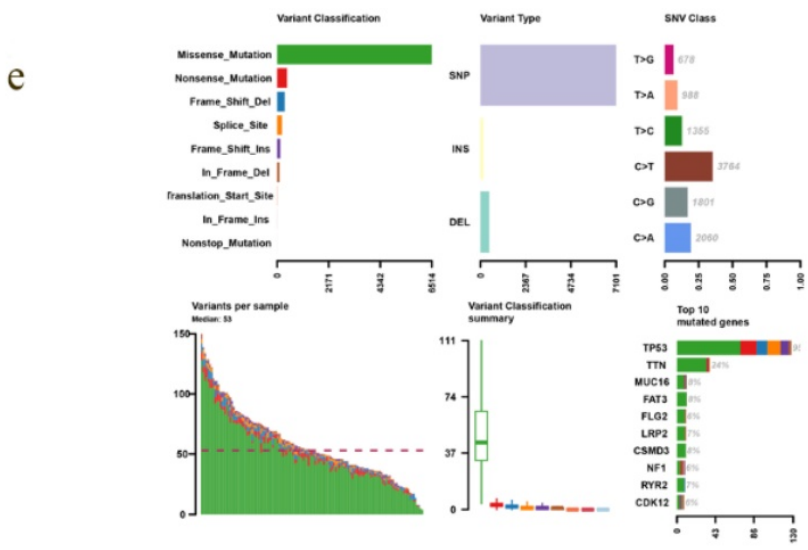

d
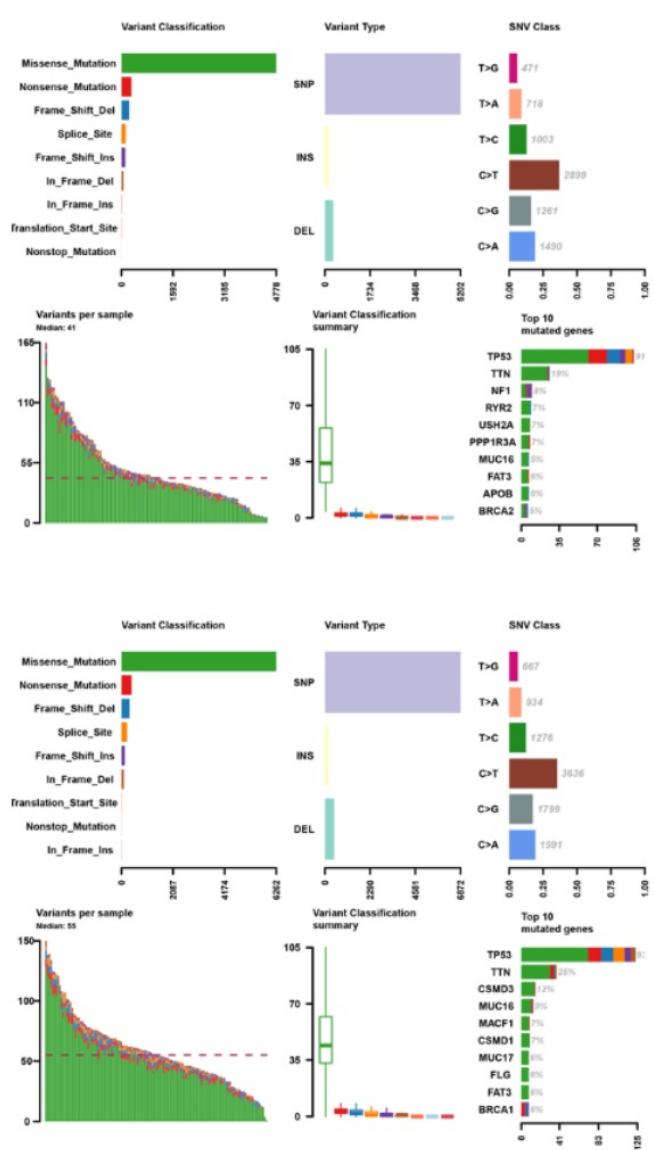

Figure 8. TMB level and mutation profiling in EOCs with different prediction of survival. a-b TMB level of low-risk group was significantly higher in both OS $(P=0.011)$ and $D F S$ model $(P=9.141$ e-04). c, e Mutation profiling of EOCs with high- and low-risk in OS model, respectively. $\mathbf{d}$, $\mathbf{f}$ Mutation profiling of cases with high- and low-risk in DFS model, respectively.

\section{Mutation profiles of high- and low-risk group}

A positive relationship between $\mathrm{TMB}$ and immunotherapy responsiveness had been reported in non-small cell lung cancer, melanoma, bladder and breast cancer patients [17-19]. Our study demonstrated that TMB correlated closely with the survival outcomes of EOC. Therefore, we compared the TMB profiles in low- and high-risk group to further verify the relationship between TMB and prognosis. Interestingly, TMB level of low-risk group was significantly higher in both OS $(P=0.011)$ and DFS model $(P=9.141 \mathrm{e}-04)$ (Fig. 8a, b), with the top 4 mutated genes including TP53, TTN, CSMD3 and MUC16 (Fig. 8e, f).

\section{Discussion}

A molecular marker-based approach to accurately predict outcomes of EOC patients is urgently needed in the era of precision medicine. TMB has been confirmed closely linked to the prognosis of cancer patients. Federico et al. [20] demonstrated that 
low TMB was one of the negative prognostic factors in patients with metastatic colorectal cancer treated in first line. Zhang et al. [8] reported that molecular subtyping based on TMB is a potential prognostic marker for lung adenocarcinoma. Nicolai et al. [21] found that TMB coupled with BRCA1 or BRCA2 mutations in ovarian cancer was a genomic marker of prognosis and predictor of treatment response. Similarly, based on the results of survival analysis, our study showed that patients in high-TMB group had better survival outcomes than those in low-TMB group, and DEGs between the two groups were identified for deep investigation.

Multiple biostatistics methods were applied to select the prognostic genes from TRGs and construct signatures to improve outcomes prediction. Seven OS-related genes (B3GALT1, LIN7B, ANGPT2, D2HGDH, TAF13, PFDN4, DNAJC19) and 4 DFS-related genes (LPIN3, PXYLP1, IGSF23, $B 3 G A L T 1)$ were detected as independent prognosis predictors in EOC. B3GALT1, the common gene of the prognostic signatures, is a member of the beta-1,3galactosyltransferase ( $\beta 3 \mathrm{GalT}$ ) gene family, which encodes type II membrane-bound glycoproteins and plays an essential role in the O-glycosylation process. Since glycoproteins rich in O-glycosylation domains are expressed on the surface of cancer cells, $\beta 3 \mathrm{GalT}$ family may be closely related to tumors [22]. Chachadi et al. [23] confirmed that B3GALT1 participated in the synthesis of Sialyl Lewis antigens, which were involved in metastasis of prostatic cancer cells. ANGPT2, also named angiopoietin-2 (Ang-2), was shown to be correlated with poor prognosis in breast, hepatocellular, colorectal and prostate cancer [24-27]. Prefoldin 4 (PFDN4) is a transcriptional factor that regulates the cell cycle, further, Miyoshi et al. [28] revealed that patients with high expression of PFDN4 showed a better prognosis for overall survival in colorectal cancer. Yet, for other genes, there are few studies about their roles in tumor prognosis.

Our study showed that patients in low-risk group had better OS and DFS than those with high risk scores in both training and entire cohort. Hence, these 2 TRG signatures were of prognostic significance. AUC values of the signatures at 1 year-, 3 years-, and 5 years in training and entire cohorts ranged from 0.608 to 0.778 , thus, large-sample researches are needed for further verification and modification before clinical application.

Functional enrichment analysis demonstrated that immune-related pathways were enriched in lowrisk groups. Among the differently infiltrated immune cells, Tfh and M1 macrophage infiltration was found to be correlated with favorable survival outcomes, while M0 macrophage correlated with poor survival in EOC cases. Similar results were demonstrated in other tumors, Tfh-related cells had been positively associated with long-term survival of humans with breast and colorectal cancer $[29,30]$. In addition, Shane Crotty [31] suggested the hypotheses for the value of Tfh cells in immune responses against tumors: (1) Tfh may help develop or support ectopic lymphoid structures, which are a site of recruitment for CD8+ T cells, NK cells, and macrophages that engage in anti-tumor immunity. Alternatively, (2) Tfh cells may support anti-tumor Ab responses by B cells. Macrophages are one of the most abundant immune cells in TME of solid tumors and their presence correlates with reduced survival in most cancers [32]. High population of macrophages is associated with poor prognosis in ovarian cancer and could impact the efficacy of immune therapies [33, 34]. Macrophages display a high plasticity, and adapt their phenotype in response to different environmental stimuli. The density of M1 macrophage in tumor islets was found to be positively associated with survival time in non-small cell lung cancer patients [35]. Interferon- $\gamma$ could induce the differentiation of M1 macrophages, and the M1 macrophages produce high levels of interleukin (IL)-12, IL-23, TNF-a, IL-1, IL-6, reactive oxygen and nitrogen intermediates, as well as other effector molecules [36-38]. Therefore, we hypothesized that the enriched "interferon response", "IL-6 signaling" and "reactive oxygen species pathway" in low-risk groups may related to the infiltration of M1 macrophages.

Usually, the complexity of tumor-infiltrating immune cells (TICs) broadly affects the host immune status and plays an important role in the response to immunotherapy. Previous studies have discovered that immune checkpoint modulators could mediate the function of TICs [39]. In our study, we found that Tfh cell and M1 macrophage correlated positively with most modulators, while M0 macrophage did negatively. And high expression of most modulators was associated with favorable outcomes in EOC. Hence, we speculated that there was an immunogenic TME in low-risk samples, which may be one of the reasons for better survival. The speculation was further confirmed by the high expression of modulators in low-risk groups.

Higher TMB level correlated with improved OS and DFS, meanwhile, TMB levels of low-risk patients were significantly higher in both OS and DFS model. Therefore, we regarded TMB as a good prognostic marker for EOC. Since TMB is positively correlated with the response to immunotherapy, and there could be an immunogenic TME in low-risk samples, we 
deduced that immunoregulation may be involved in the prognosis prediction of TMB in EOC.

Then, we further explored the TMB profiles, and found TP53, TTN, FAT3, CSMD3, MUC16 and FLG were among the top 10 mutated genes of low-risk group in both OS and DFS model. The most predominant genetic alternation in EOC is the mutation of TP53, which had been considered as diagnostic and prognostic biomarker as well as therapeutic target for EOC [40]. Wieser et al. [41] found that TP53 mutation might serve as a potential predictive factor of anti-PD1/PD-L1 immunotherapy in ovarian cancer. MUC16 encodes a repeating peptide epitope of mucin that promotes cancer cell proliferation and inhibits anti-cancer immune responses [42]. Filaggrin (FLG), was found to identify a distinct patient population with low immune cell infiltration in melanoma and ovarian cancer, and may protect cancer cells from immune cell infiltration and immune-mediated destruction [43]. Mutations of FAT3, CSMD3 and TTN have been reported in EOC $[44,45]$, but their influence on immunoregulation was still unknown.

However, there are also some limitations to our study: (i) basic experiment is needed to validate the association between genes signatures and immune infiltrates; (ii) lack of external validation; (iii) further studies with lager sample size are required in the future.

\section{Conclusion}

We constructed and validated 2 TMB-related prognostic signatures, which may act as promising prognostic molecular biomarkers and have immunotherapeutic implications for EOC patient management.

\section{Supplementary Material}

Supplementary figures and tables.

http://www.medsci.org/v17p3200s1.pdf

\section{Acknowledgments}

\section{Funding}

This work was supported by the National Nature Science Foundation of China (81872119 and 81472442).

\section{Author contributions}

JL and WX conceived and designed the study. JL obtained the dataset and conducted data analysis. WX wrote the manuscript and SL completed the figures. WC revised the manuscript. All authors approved the final version of the manuscript.

\section{Competing Interests}

The authors have declared that no competing interest exists.

\section{References}

1. Oronsky B, Ray CM, Spira AI, et al.. A brief review of the management of platinum-resistant-platinum-refractory ovarian cancer. Med Oncol. 2017; 34: 103.

2. Tsibulak I, Zeimet AG, Marth C. Hopes and failures in front-line ovarian cancer therapy. Crit Rev Oncol Hematol. 2019; 143: 14-19.

3. Disis ML, Taylor MH, Kelly K, et al.. Efficacy and Safety of Avelumab for Patients With Recurrent or Refractory Ovarian Cancer: Phase 1b Results From the JAVELIN Solid Tumor Trial. JAMA Oncol. 2019; 5: 393-401.

4. Hamanishi J, Mandai M, Ikeda T, et al.. Safety and Antitumor Activity of Anti-PD-1 Antibody, Nivolumab, in Patients With Platinum-Resistant Ovarian Cancer. J Clin Oncol. 2015; 33: 4015-4022.

5. Inayama Y, Hamanishi J, Matsumura N, et al.. Antitumor Effect of Nivolumab on Subsequent Chemotherapy for Platinum-Resistant Ovarian Cancer. Oncologist. 2018; 23: 1382-1384.

6. Brahmer JR, Tykodi SS, Chow LQ, et al.. Safety and activity of anti-PD-L1 antibody in patients with advanced cancer. N Engl J Med. 2012; 366: 2455-2465.

7. Marth C, Wieser V, Tsibulak I, et al.. Immunotherapy in ovarian cancer: fake news or the real deal? Int J Gynecol Cancer. 2019; 29: 201-211.

8. Wang $\mathrm{C}$, Liang $\mathrm{H}$, Lin $\mathrm{C}$, et al.. Molecular Subtyping and Prognostic Assessment Based on Tumor Mutation Burden in Patients with Lung Adenocarcinomas. Int J Mol Sci. 2019; 20: 17.

9. Rizvi NA, Hellmann MD, Snyder A, et al.. Mutational landscape determines sensitivity to PD-1 blockade in non-small cell lung cancer. Science. 2015; 348: 124-128.

10. Snyder A, Makarov V, Merghoub T, et al.. Genetic basis for clinical response to CTLA-4 blockade in melanoma. N Engl J Med. 2014; 371: 2189-2199.

11. Morse CB, Elvin JA, Gay LM, et al.. Elevated tumor mutational burden and prolonged clinical response to anti-PD-L1 antibody in platinum-resistant recurrent ovarian cancer. Gynecol Oncol Rep. 2017; 21: 78-80.

12. Liu $\mathrm{X}, \mathrm{Wu} \mathrm{S}$, Yang $\mathrm{Y}$, et al.. The prognostic landscape of tumor-infiltrating immune cell and immunomodulators in lung cancer. Biomed Pharmacother. 2017; 95: 55-61.

13. Lim S, Phillips JB, Madeira da Silva L, et al.. Interplay between Immune Checkpoint Proteins and Cellular Metabolism. Cancer Res. 2017; 77: 1245-1249.

14. Preusser M, Lim M, Hafler DA, et al.. Prospects of immune checkpoint modulators in the treatment of glioblastoma. Nat Rev Neurol. 2015; 11: 504-514.

15. Vesely MD, Schreiber RD. Cancer immunoediting: antigens, mechanisms, and implications to cancer immunotherapy. Ann N Y Acad Sci. 2013; 1284: 1-5.

16. Wang J, Li Z, Gao A, et al.. The prognostic landscape of tumor-infiltrating immune cells in cervical cancer. Biomedicine \& Pharmacotherapy. 2019; 120: 109444.

17. Heeke S, Benzaquen J, Long-Mira E, et al.. In-house Implementation of Tumor Mutational Burden Testing to Predict Durable Clinical Benefit in Non-small Cell Lung Cancer and Melanoma Patients. Cancers (Basel). 2019; 11: 9.

18. Zhang C, Shen L, Qi F, et al.. Multi-omics analysis of tumor mutation burden combined with immune infiltrates in bladder urothelial carcinoma. J Cell Physiol. 2020; 235: 3849-3863.

19. Thomas A, Routh ED, Pullikuth A, et al.. Tumor mutational burden is a determinant of immune-mediated survival in breast cancer. Oncoimmunology. 2018; 7: e1490854.

20. Innocenti $\mathrm{F}, \mathrm{Ou}$ FS, $\mathrm{Qu} \mathrm{X}$, et al. Mutational Analysis of Patients With Colorectal Cancer in CALGB/SWOG 80405 Identifies New Roles of Microsatellite Instability and Tumor Mutational Burden for Patient Outcome. J Clin Oncol. 2019; 37: 1217-1227.

21. Birkbak NJ, Kochupurakkal B, Izarzugaza JM, et al.. Tumor mutation burden forecasts outcome in ovarian cancer with BRCA1 or BRCA2 mutations. PLoS One. 2013; 8: e80023.

22. Wandall $\mathrm{HH}$, Blixt $\mathrm{O}$, Tarp $\mathrm{MA}$, et al.. Cancer biomarkers defined by autoantibody signatures to aberrant O-glycopeptide epitopes. Cancer Res. 2010; 70: 1306-1313.

23. Chachadi VB, Ali MF, Cheng PW. Prostatic cell-specific regulation of the synthesis of MUC1-associated sialyl Lewis a. PLoS One. 2013; 8: e57416.

24. Sfiligoi C, de Luca A, Cascone I, et al.. Angiopoietin-2 expression in breast cancer correlates with lymph node invasion and short survival. Int J Cancer. 2003; 103: 466-474.

25. Mitsuhashi N, Shimizu H, Ohtsuka M, et al.. Angiopoietins and Tie-2 expression in angiogenesis and proliferation of human hepatocellular carcinoma. Hepatology. 2003; 37: 1105-1113.

26. Ogawa M, Yamamoto H, Nagano H, et al.. Hepatic expression of ANG2 RNA in metastatic colorectal cancer. Hepatology. 2004; 39: 528-539.

27. Lind AJ, Wikstrom P, Granfors T, et al.. Angiopoietin 2 expression is related to histological grade, vascular density, metastases, and outcome in prostate cancer. Prostate. 2005; 62: 394-399. 
28. Miyoshi N, Ishii $\mathrm{H}$, Mimori $\mathrm{K}$, et al.. Abnormal expression of PFDN4 in colorectal cancer: a novel marker for prognosis. Ann Surg Oncol. 2010; 17: 3030-3036.

29. Bindea G, Mlecnik B, Tosolini M, et al.. Spatiotemporal dynamics of intratumoral immune cells reveal the immune landscape in human cancer. Immunity. 2013; 39: 782-795.

30. Gu-Trantien C, Loi S, Garaud S, et al.. CD4(+) follicular helper T cell infiltration predicts breast cancer survival. J Clin Invest. 2013; 123: 2873-2892.

31. Crotty S. T Follicular Helper Cell Biology: A Decade of Discovery and Diseases. Immunity. 2019; 50: 1132-1148.

32. Nielsen SR, Schmid MC. Macrophages as Key Drivers of Cancer Progression and Metastasis. Mediators Inflamm. 2017; 2017: 9624760

33. Zhang $\mathrm{M}, \mathrm{He} \mathrm{Y}$, Sun $\mathrm{X}$, et al.. A high M1/M2 ratio of tumor-associated macrophages is associated with extended survival in ovarian cancer patients. J Ovarian Res. 2014; 7: 19.

34. Yuan X, Zhang J, Li D, et al.. Prognostic significance of tumor-associated macrophages in ovarian cancer: A meta-analysis. Gynecol Oncol. 2017; 147: 181-187.

35. Ma J, Liu L, Che G, et al.. The M1 form of tumor-associated macrophages in non-small cell lung cancer is positively associated with survival time. BMC Cancer. 2010; 10: 112.

36. Mantovani A, Sica A, Locati M. New vistas on macrophage differentiation and activation. Eur J Immunol. 2007; 37: 14-16.

37. Mantovani A, Sica A, Locati M. Macrophage polarization comes of age. Immunity. 2005; 23: 344-346.

38. Mantovani A, Sozzani S, Locati M, et al.. Macrophage polarization: tumor-associated macrophages as a paradigm for polarized M2 mononuclear phagocytes. Trends Immunol. 2002; 23: 549-555.

39. Santoiemma PP, Powell DJ, Jr. Tumor infiltrating lymphocytes in ovarian cancer. Cancer Biol Ther. 2015; 16: 807-820.

40. Zhang Y, Cao L, Nguyen D, et al.. TP53 mutations in epithelial ovarian cancer. Translational Cancer Research. 2016; 5: 650-663.

41. Wieser V, Gaugg I, Fleischer M, et al.. BRCA1/2 and TP53 mutation status associates with PD-1 and PD-L1 expression in ovarian cancer. Oncotarget. 2018; 9: 17501-17511.

42. Comamala M, Pinard M, Theriault C, et al.. Downregulation of cell surface CA125/MUC16 induces epithelial-to-mesenchymal transition and restores EGFR signalling in NIH: OVCAR3 ovarian carcinoma cells. Br J Cancer. 2011; 104: 989-999.

43. Salerno EP, Bedognetti D, Mauldin IS, et al.. Human melanomas and ovarian cancers overexpressing mechanical barrier molecule genes lack immune signatures and have increased patient mortality risk. Oncoimmunology. 2016; 5: e1240857.

44. Zhang L, Luo M, Yang H. Next-generation sequencing-based genomic profiling analysis reveals novel mutations for clinical diagnosis in Chinese primary epithelial ovarian cancer patients. J Ovarian Res. 2019; 12: 19.

45. Longacre M, Snyder NA, Housman G, et al.. A Comparative Analysis of Genetic and Epigenetic Events of Breast and Ovarian Cancer Related to Tumorigenesis. Int J Mol Sci.2016; 17: 5. 